\title{
Closing in on minimal dark matter and radiative neutrino masses
}

\section{Aristizabal Sierra, ${ }^{a, b}$ C. Simoes ${ }^{a}$ and D. Wegman ${ }^{a}$}

${ }^{a}$ IFPA, Dép. AGO, Université de Liège, Bât B5, Sart Tilman B-4000 Liège 1, Belgium

${ }^{b}$ Departamento de Física, Universidad Técnica Federico Santa María, Casilla 110-V, Avda. España 1680, Valparaiso, Chile

E-mail: daristizabal@ulg.ac.be, csimoes@ulg.ac.be, dwegman@ulg.ac.be

ABSTRACT: We study one-loop radiative neutrino mass models in which one of the beyondthe-standard model fields is either a hypercharge-zero fermion quintet (minimal dark matter) or a hypercharge-zero scalar septet. By systematically classifying all possible one-loop such models we identify various processes that render the neutral component of these representations (dark matter) cosmologically unstable. Thus, our findings show that these scenarios are in general not reconcilable with dark matter stability unless tiny couplings or additional ad hoc symmetries are assumed, in contrast to minimal dark matter models where stability is entirely due to the standard model gauge symmetry. For some variants based on higher-order loops we find that $\alpha_{2}$ reaches a Landau pole at rather low scales, a couple orders of magnitude from the characteristic scale of the model itself. Thus, we argue that some of these variations although consistent with dark matter stability and phenomenological constraints are hard to reconcile with perturbativity criteria.

Keywords: Beyond Standard Model, Neutrino Physics

ArXIV EPRINT: 1603.04723 


\section{Contents}

1 Introduction 1

2 Minimal dark matter and possible decay processes 3

2.1 Minimal DM decay in the presence of extra degrees of freedom 5

3 UV completions of one-loop realizations of the Weinberg operator $\quad 10$

$\begin{array}{ll}3.1 \text { The hypercharge-zero quintet case } & 10\end{array}$

$\begin{array}{lll}3.2 & \text { The hypercharge-zero septet case } & 12\end{array}$

4 Pitfalls of DM from higher-order representations and neutrino masses 13

5 Conclusions

\section{Introduction}

Neutrino physics, dark matter (DM) and the cosmic baryon asymmetry provide strong evidence for beyond-the-Standard Model (BSM) physics. Neutrino masses and their mixing pattern can be well-described at the effective level by a certain lepton-number-violating operator [1], with the $\operatorname{dim}=5$ being the obvious choice. Conventional wisdom is that this operator originates from a type-I seesaw involving a GUT scale. The reason for this is probably related with the fact that such picture nicely fits within $\mathrm{SO}(10)$-inspired scenarios, while at the same time explains the absence of charged lepton-flavor-violating signals in high-intensity experiments [2]..$^{1}$ In contrast to neutrino physics, there is no such "standard" scenario for DM, and several phenomenologically-motivated frameworks exist [4]. However, of particular popularity are WIMP scenarios where the DM relic density results from thermal freeze-out.

Other well-motivated scenarios for neutrino mass generation exist and provide a potential link between the origin of neutrino masses and that of DM [5] (see e.g. [6] for a phenomenological analysis). This connection somehow resembles what one finds in the type-I seesaw, where neutrino masses and the cosmic baryon asymmetry find a common explanation through standard leptogenesis (see $[7,8]$ for reviews). ${ }^{2}$ Particularly relevant for that endeavor are models where the $\operatorname{dim}=5$ lepton-number-violating operator arises through radiative corrections (see $[11,12]$ for a full list of all such possibilities at the oneand two-loop level). In these contexts several approaches have been adopted to assure DM stability. The conventional one relies on the introduction of ad hoc symmetries under

\footnotetext{
${ }^{1}$ Seesaw-like scenarios with sizeable lepton-flavor-violating effects can be constructed, but they require deviations from the "standard" type-I seesaw picture, see e.g. [3].

${ }^{2}$ Variants of the standard leptogenesis picture can be embedded within type-I seesaw or in other neutrino mass models as well, see e.g. [9, 10].
} 
which the SM and the dark sector transform differently, thus guaranteeing stability. These symmetries can have multiple origins, and can be regarded as remnants of the spontaneous symmetry breaking of a more fundamental symmetry related with e.g. GUTs $[13,14]$ or flavor theories [15].

Another approach that has been explored relates radiative neutrino masses with higherorder electroweak (EW) representations [16-24]. In this case the idea is different and relies on the mechanism underlying minimal DM models [25-27], namely for higher-order EW representations renormalizable interactions with SM operators cannot be written, and so they are absolutely stable at the renormalizable level (at the renormalizable level the minimal DM Lagrangian exhibits an accidental $\mathbb{Z}_{2}$ symmetry). Decays are induced by effective operators, thus in these scenarios DM is never absolutely stable but can be cosmologically stable provided the effective operator is sufficiently suppressed. In [25] it was shown that the stability condition, the requirement of EW gauge coupling perturbativity up to $M_{\text {Planck }}$ and direct DM searches constraints, singles out two possible representations: hyperchargezero fermion quintet and hypercharge-zero scalar septet, of which the fermion quintet defines the minimal DM model. Recently, in refs. [28] and subsequently in [29] it was shown that for the scalar septet one can write a loop-induced lower dimensional operator that renders the septet a non-viable DM representation, even if the effective scale is assumed to be $M_{\text {Planck }}$. Thus, within the minimal DM context the only viable representation is the fermion quintet, although recently analyses have shown that depending on the DM profile this representation is not consistent with indirect DM searches results [30, 31] (see discussion in section 2).

In this paper we show that when the fermion quintet acts as a mediator in one-loop neutrino mass generation, there are always DM decay operators that - under reasonable parameter choices - lead to fast DM decays, regardless of the one-loop neutrino mass model. Some of these operators arise at the tree level but others are loop-induced. The latter being specially important in models where in addition to the fermion quintet, representations beyond quartets are present. We will show that as soon as the minimal DM scheme is extended to include other representations (that allow the construction of one-loop neutrino mass models) there are always $\mathbb{Z}_{2}$-breaking couplings, thus fast decay modes are always expected to be present. We consider the case of the scalar septet as well, despite being not consistent with stability even in the minimal DM framework. The reason is that these results allow the identification of DM fast decay modes induced by neutrino physics itself, rather than due to a different type of physics (related with unknown quantum gravity effects).

The rest of this paper is organized as follows. In section 2, we briefly review minimal DM models, introduce our notation and present formulas that will be used in our estimations. In section 2.1 , we present the different $\mathbb{Z}_{2}$-breaking decay operators and estimate lifetimes for various processes, showing that even for neutrino physics parameters taken to their extreme values the decays are always fast. In section 3, we systematically derive all one-loop realizations of the Weinberg operator, under the condition of the UV completion containing either the fermion quintet or scalar septet. With these results at hand, we then identify the different DM decay operators associated with each of the neutrino mass 
model categories. In section 4, we discuss the main pitfalls of models for Majorana neutrino masses with higher-order EW representations and briefly comment on models with higher-order loops. Finally, in section 5 we summarize and present our conclusions.

\section{Minimal dark matter and possible decay processes}

Minimal DM models rely on the observation that for higher-order SU(2) representations, $\boldsymbol{R}$, renormalizable gauge invariant operators of the form

$$
\mathcal{O}_{N=4} \sim \boldsymbol{R} \mathcal{O}_{\mathrm{SM}}
$$

where $\mathcal{O}_{\mathrm{SM}}$ is an operator involving just SM fields, cannot be written [25-27]. Thus, when the SM is endowed with such state, its stability is automatically guaranteed. The stabilization mechanism at work, resembles the SM "mechanism" which assures proton stability, namely the gauge symmetry does not allow for renormalizable operators that might induce decay. Of course, at the non-renormalizable level several effective operators of the form (2.1) can be written, but their renormalizable realizations require degrees of freedom which the minimality criteria do not allow for. ${ }^{3}$

The lowest-order representation for which the above argument proves to be true and yields a viable DM candidate is the fermionic $\boldsymbol{R}=\mathbf{5}$. Let us discuss this in more detail. For $\boldsymbol{R}=\mathbf{3}$, renormalizable operators can always be written, regardless of whether they are fermions $(F)$ or scalars $(S)$ and provided $Y=0,1$. For the quartet and beyond it is more subtle. Using the notation $\boldsymbol{R}_{X}^{Y}$ (with $X$ referring to fermion $(F)$ or scalar $(S)$ and $Y$ to hypercharge, normalized according to: $Q=T_{3}+Y$ ), and bearing in mind that

$$
\underbrace{\mathbf{2} \otimes \cdots \otimes 2}_{(R-1) \text { times }} \supset \boldsymbol{R}
$$

one finds that for the quartet non-renormalizable operators are possible depending on its spin, namely

$$
\begin{aligned}
& \mathcal{O}_{N=5} \sim \mathbf{4}_{F}^{Y} \mathcal{O}_{\mathrm{SM}}\left\{\begin{array}{ll}
\mathcal{O}_{\mathrm{SM}}=\ell H H^{\dagger} & (Y=1 / 2) \\
\mathcal{O}_{\mathrm{SM}}=\ell H^{\dagger} H^{\dagger} & (Y=3 / 2)
\end{array},\right. \\
& \mathcal{O}_{N=4} \sim \mathbf{4}_{S}^{Y} \mathcal{O}_{\mathrm{SM}}\left\{\begin{array}{ll}
\mathcal{O}_{\mathrm{SM}}=H^{\dagger} H H^{\dagger} & (Y=1 / 2) \\
\mathcal{O}_{\mathrm{SM}}=H^{\dagger} H^{\dagger} H^{\dagger} & (Y=3 / 2)
\end{array} .\right.
\end{aligned}
$$

However, though involving non-renormalizable operators, $\boldsymbol{R}_{F}=\mathbf{4}_{F}$ does not provide a viable DM candidate (the $\boldsymbol{R}_{S}=\boldsymbol{4}_{S}$ involves renormalizable couplings and so it is of course non-viable either). First of all, even if the cutoff scale is taken to be $\Lambda=M_{\text {Planck }}$ (where arguably quantum gravity effects will generate such operator) its suppression is not sufficiently strong to guarantee cosmological stability. Furthermore, in the context of minimal DM only $Y=0$ representations are consistent with direct DM searches: for $Y \neq 0$ states,

\footnotetext{
${ }^{3}$ Something of this sort is found in the SM as well. Baryon- and lepton-number-violating effective interactions can be written $[1,32]$, but their renormalizable forms require beyond-the-SM physical states.
} 
$Z$-mediated processes induce a tree-level spin-independent DM-nucleon cross section well above the values allowed by current DM direct searches [25-27]. We stress that the validity of this statement is subject to the minimality condition. Departures from this assumption, e.g. by introducing another state that mixes with the DM producing a small mass splitting, enable $Y \neq 0$ representations (for more detailes see [27]). For $\boldsymbol{R}_{F}=\mathbf{5}_{F}$ one finds $\mathbf{2} \otimes \mathbf{2} \otimes \mathbf{2} \otimes \mathbf{2} \supset \mathbf{5}$, which then implies that SU(2) invariance requires four SM fields. Depending on the spin of the representation, the effective operator in (2.1) is $N=5$ (scalar) or $N=6$ (fermion), with its full structure determined by the hypercharge of the corresponding state.

An upper limit on the size of the representations can be determined by the condition of $\alpha_{2}$ being perturbative up to the Planck scale. Ref. [28] has shown that depending on the representation a precise determination of the Landau pole should rely on two-loop RGE. Based on that analysis representations $\boldsymbol{R}_{F}>\mathbf{5}_{F}$ and $\boldsymbol{R}_{S}>\boldsymbol{8}_{S}$ are ruled out, as has been previously pointed out in [25-27] using a one-loop RGE calculation. One is left then with $\boldsymbol{R}<\mathbf{5}_{F, S}$ and $\mathbf{7}_{S}$ for which the decay lifetimes can be estimated from the effective operator

$$
\mathcal{O}_{N}=\frac{c_{N}}{\Lambda^{N-4}} \boldsymbol{R} \mathcal{O}_{\mathrm{SM}}
$$

where the Wilson coefficient $c_{N}$, assumed to be order one for simplicity, can involve flavor indices too. In the limit $m_{\mathrm{DM}} \gg v$ (with $v=\langle H\rangle=\left(\sqrt{2} G_{F}\right)^{-1 / 2} \simeq 246 \mathrm{GeV}$ ), the total decay width can be parameterized as follows [28]

$$
\Gamma_{\mathrm{DM}}^{(N)}=\frac{1}{4(4 \pi)^{2 N-5}} \frac{m_{\mathrm{DM}}^{2 N-2 n_{c}-7}}{(N-2) !(N-3) !} \frac{(v / \sqrt{2})^{2 n_{c}}}{\Lambda^{2(N-4)}},
$$

where $n_{c}$ refers to the number of Higgs condensate insertions. Of the three potentially viable representations only those for which $\tau_{\mathrm{DM}} \gtrsim 10^{26} \mathrm{sec}$ (as required by indirect detection experiments looking for $\gamma$-ray, $\nu, e^{+}$or $p^{-}$signals stemming from DM decay [33-36]) are consistent. From (2.4) their lifetimes can be calculated. Consistency with $\tau_{\mathrm{DM}} \gtrsim 10^{26} \mathrm{sec}$ for $\mathbf{5}_{S}^{0}$ requires scales way above $M_{\text {Planck }}$, in contrast $\mathbf{5}_{F}^{0}$ and $\mathbf{7}_{S}^{0}$ have sufficiently long lifetimes even for scales well below the Planck scale, due to their decays being driven by $\operatorname{dim}=6$ and $\operatorname{dim}=7$ operators as can be seen in figure 1.

In the absence of any other degree of freedom, these results hold if no lower dimension operator for these representation can be written. For $\mathbf{5}_{F}^{0}$ this proves to be true, for $\mathbf{7}_{S}^{0}$, instead, not. The point is that the previous analysis rely on tree level effective decay modes, but for $7_{S}^{0}$ one-loop radiative dim $=5$ operators can be written through the operator $\mathbf{7}_{S}^{0}\left(\mathbf{7}_{S}^{0} \boldsymbol{7}_{S}^{0}\right) H^{\dagger} H H^{\dagger} H$ [28] (see discussion below). Thus, this argument rules out the $\mathbf{7}_{S}^{0}$ as well. Minimal DM therefore reduces to a single possibility, $\mathbf{5}_{F}^{0}$, for which stringent constraints from indirect DM searches have been found. Of particular relevance are those coming from $\gamma$-ray line searches from the galactic center, for which it has been proven that if the Milky Way possesses a Navarro-Frenk-White or Einasto DM profiles this representation is ruled out too $[30,31] .{ }^{4}$ It can be however consistently considered in the context of cored profiles such as Burket or Isothermal.

\footnotetext{
${ }^{4}$ We thank Thomas Hambye for pointing this out to us.
} 


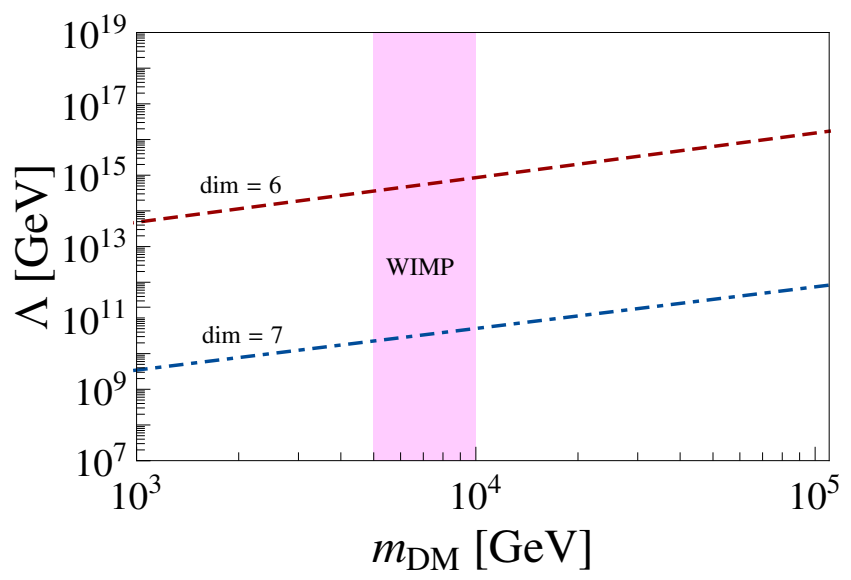

Figure 1. Contour lines of constant $\tau_{\mathrm{DM}}=10^{26}$ seconds as a function of the cutoff scale $\Lambda$ and $m_{\mathrm{DM}}$. From top to bottom the contour lines refer to dimension 6 and 7 effective operators that induce DM decay. The vertical stripe encloses the DM mass region where the DM relic density results from thermal freeze-out.

\subsection{Minimal DM decay in the presence of extra degrees of freedom}

Minimal DM instability is induced by UV completions associated with effective operators of the type (2.3). In the minimal model the new degrees of freedom are assumed to be related with quantum gravity effects, something that guarantees stability for $\mathbf{5}_{F}^{0}$. For UV completions associated with "lighter" physics, DM can become cosmologically unstable. For $\Lambda \lesssim 10^{15} \mathrm{GeV}, \mathbf{5}_{F}^{0}$ has a lifetime below $10^{26}$ sec.

The neutrino mass one gets from one-loop realizations of the Weinberg operator can be estimated as

$$
m_{\nu} \sim \frac{v^{2}}{16 \pi^{2}} \frac{Y^{4}}{\Lambda}
$$

where $Y$ denotes a generic Yukawa coupling. Thus, assuming $Y \subset\left[10^{-2}, 1\right]$ and fixing the neutrino mass to $m_{\mathrm{Atm}}=0.05 \mathrm{eV}[37-39]$, one can estimate $\Lambda \lesssim\left[10^{5}, 10^{13}\right] \mathrm{GeV}$. Note that in this estimation the one-loop function has been neglected, but in a specific model with an explicit neutrino mass matrix the presence of the loop function will lead to smaller cutoff scales. Thus, with the value derived from (2.5) we are overestimating the DM lifetime.

This result then shows that - in principle - any one-loop UV completion of the Weinberg operator that can yield in turn an UV completion for the effective operator responsible for $\mathbf{5}_{F}^{0}$ decays will lead to:

$$
\tau_{\mathrm{DM}} \lesssim 2 \times 10^{18}\left(\frac{10^{4} \mathrm{GeV}}{m_{\mathrm{DM}}}\right)^{5}\left(\frac{\Lambda}{10^{13} \mathrm{GeV}}\right)^{4} \text { sec. }
$$

and therefore to unviable "minimal DM". ${ }^{5}$ This conclusion can be understood as follows. In the absence of further degrees of freedom the minimal DM full Lagrangian reads:

$$
\mathcal{L}=\mathcal{L}_{\mathrm{SM}}+\operatorname{Tr}\left[\overline{\overline{\mathbf{5}}_{F}} i \not D \mathbf{5}_{F}\right]+\frac{1}{2} m_{5}\left(\overline{\mathbf{5}_{F}^{c}} \mathbf{5}_{F}+\text { H.c. }\right) .
$$

\footnotetext{
${ }^{5}$ Strictly speaking once minimal DM is embedded in radiative neutrino mass models (or any other scenario) is not anymore minimal DM since its defining conditions do not hold anymore.
} 
This Lagrangian is invariant under the $\mathbb{Z}_{2}$ transformations $\mathbf{5}_{F} \rightarrow-\mathbf{5}_{F}$ and $X_{\mathrm{SM}} \rightarrow X_{\mathrm{SM}}$, that entirely results as a consequence of the gauge symmetry. If the extra representations that allow for the one-loop neutrino mass matrix allow as well for UV completions of the following Lagrangian

$$
\mathcal{L}_{\text {eff }}=\frac{c_{6}}{\Lambda^{2}} \mathbf{5}_{F} \ell H H^{\dagger} H
$$

they will necessarily involve interactions that explicitly break the accidental $\mathbb{Z}_{2}$ symmetry (as this operator does) and therefore will lead to DM decay processes with typical lifetimes given by (2.6). Instability of the $\mathbf{7}_{S}^{0}$ can be understood in the same way. The full Lagrangian is given by

$$
\mathcal{L}=\left|D_{\mu} \mathbf{7}_{S}\right|^{2}+V_{\mathrm{SM}}+m_{7}^{2}\left|\mathbf{7}_{S}\right|^{2}+\lambda_{1}\left|\mathbf{7}_{S}\right|^{2}|H|^{2}+\cdots,
$$

where the ellipses refer to additional terms, that as the ones we have explicitly written are $\mathbb{Z}_{2}$-invariant. In this case the relevant effective $\mathbb{Z}_{2}$-breaking term is

$$
\mathcal{L}_{\text {eff }}=\frac{c_{5}}{\Lambda} \boldsymbol{7}_{S} \mathbf{7}_{S} \mathbf{7}_{S} H^{\dagger} H .
$$

This operator alone cannot yield DM decays, but when combined with the EW invariant $H^{\dagger} H$ leads to a one-loop $\operatorname{dim}=5$ decay operator [28]. As in the fermionic DM case, instability of the $\boldsymbol{7}_{S}$ can be understood as a consequence of renormalizable $\mathbb{Z}_{2}$-violating interactions that in the effective limit reduce to (2.10).

As already pointed out, decay modes depend upon the extra representations and can be sorted according to tree level and one-loop induced decay modes. In what follows whenever calculating lifetimes we will set $m_{\mathrm{DM}}=10^{4} \mathrm{GeV}$ and the mass of the extra representations, assumed to be universal $(m)$, to $10^{9} \mathrm{GeV}$, which is typically the largest value for the extra degrees of freedom one will get in a specific neutrino mass model. With the relevant masses fixed in this way, the $\mathbb{Z}_{2}$-conserving Yukawa couplings (those related with neutrino mass generation: $Y_{\nu}$ ) are fixed to one. ${ }^{6}$ We start our analysis with the tree level modes:

- Tree level decay modes for $\mathbf{5}_{F}^{0}$. These DM decay processes are mediated by scalar or fermion $Y=1 / 2$ quartets and sextets, as required by gauge invariance. In both cases one can distinguish processes with no additional representations (single messenger scenarios) and processes with further representations, e.g. scalar triplets or any other enabled by gauge invariance (multiple messenger scenarios). In single messenger processes, DM decays proceed via off-shell states that in turn directly decay to SM particles, through either renormalizable or non-renormalizable couplings (examples are shown in figure 2, diagrams (a), (c), (d)). In multiple messenger scenarios, instead, DM decay via cascade processes mediated by several off-shell states, see figure 2 diagram (b) for an example. Note that one can as well write multiple messenger decay modes for the sextet by expanding the effective couplings $c_{6}^{S}$ and $c_{7}^{F}$ in diagrams (c) and (d) in figure 2. In what follows we discuss in more detail the examples shown in figure 2, aiming at showing that in models involving quartets tree level DM decay

\footnotetext{
${ }^{6}$ This parameter choice is a simplification that enables determining the largest lifetimes achievable in each case. In practice, however, these scales are not entirely independent and instead are constrained by phenomenological conditions such as the DM relic abundance.
} 


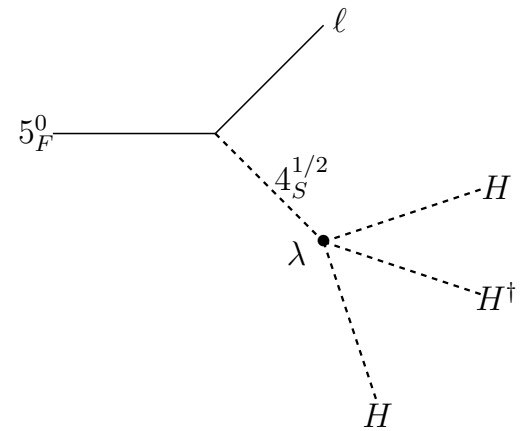

(a)

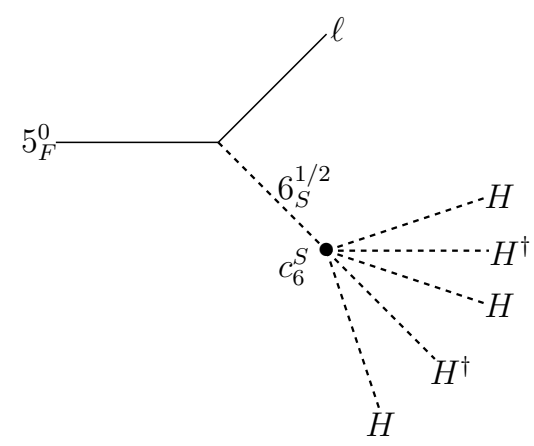

(c)

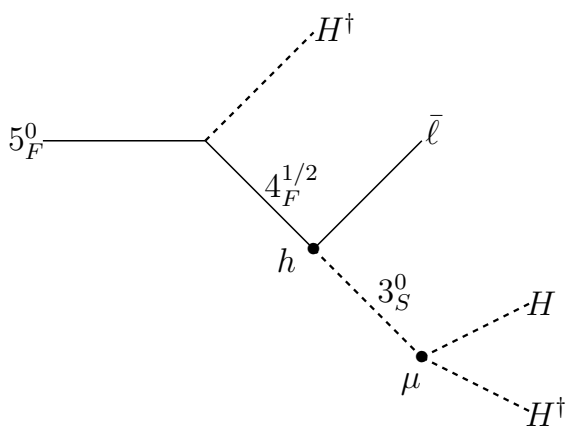

(b)

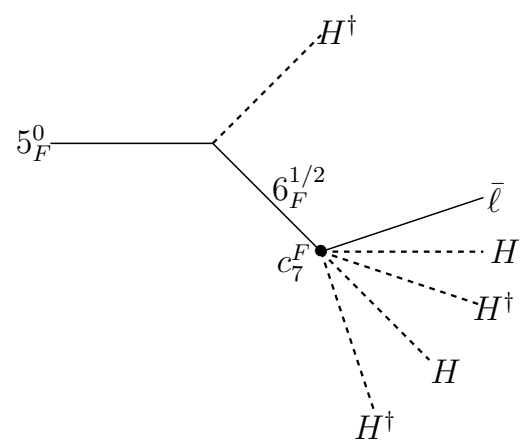

(d)

Figure 2. Upper panel: tree level operators responsible for $\chi_{\mathrm{DM}} \subset \mathbf{5}_{F}^{0}$ decay with a single (left) and multiple (right) messenger fields. Lower panel: tree level operators for $\chi_{\mathrm{DM}} \subset \mathbf{5}_{F}^{0}$ decay for messenger fields not allowing renormalizable couplings. Dots indicate $\mathbb{Z}_{2}$-breaking couplings.

processes always lead to fast DM decay, while for those with sextets these processes do not lead to rapid decay modes, therefore motivating the investigation of DM decay loop-induced processes.

Of particular relevance for one-loop neutrino mass models is $4_{S}^{1 / 2}$ for which one can write $\mathbf{5}_{F}^{0} \rightarrow \ell \mathbf{4}_{S}^{1 / 2} \rightarrow \ell H H^{\dagger} H$ (see figure 2). Depending on the different $\mathrm{SU}(2)$ contractions this operator chain contains several decay processes, of which $l^{ \pm} W_{L}^{\mp} Z_{L} Z_{L}$ is the dominant decay mode. The lifetime can be estimated by inserting $c_{6}=Y \cdot \lambda$ in eq. (2.4) and trading $\Lambda$ to $m$. The result reads:

$$
\tau_{\mathrm{DM}} \lesssim 2 \times 10^{6}\left(\frac{10^{-2}}{\lambda}\right)^{2}\left(\frac{10^{4} \mathrm{GeV}}{m_{\mathrm{DM}}}\right)^{5}\left(\frac{m}{10^{9} \mathrm{GeV}}\right)^{4} \text { sec. }
$$

which rules out the possibility of stable $\chi_{\mathrm{DM}} \subset \mathbf{5}_{S}^{0}$ in one-loop neutrino mass models that contain a $\mathbf{4}_{S}^{1 / 2}$ (see discussion in section 4 ). As an example of multiple messenger processes we consider $\mathbf{4}_{F}^{1 / 2}$ and $\mathbf{3}_{S}^{0}$, tree level decay processes associated with $\mathbf{5}_{F}^{0} \rightarrow$ $H^{\dagger} \mathbf{4}_{F}^{1 / 2} \rightarrow H^{\dagger} \bar{\ell} H H^{\dagger}$ can be written. In contrast to the single messenger case, in these scenarios as obviously expected more $\mathbb{Z}_{2}$-violating couplings are involved (see 


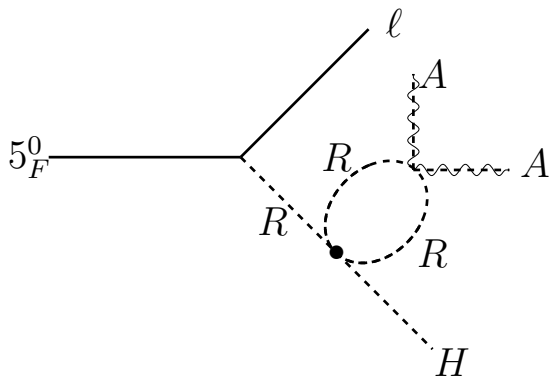

sl-i

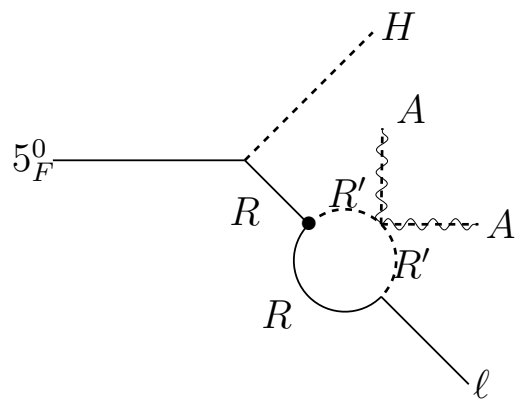

fl-i

Figure 3. One-loop diagrams responsible for $\chi_{\mathrm{DM}} \subset \mathbf{5}_{F}^{0}$ instability. Dots indicate $\mathbb{Z}_{2}$-breaking couplings. Label sl-i refers to scalar loops, while fl-i to fermion loops. For the diagram to the left $\boldsymbol{R}=\mathbf{4}_{S}^{1 / 2}$ or $\boldsymbol{R}=\mathbf{6}_{S}^{1 / 2}$ (sl-1 and sl-2, respectively), while for the diagrams to the right $\left(\boldsymbol{R}, \boldsymbol{R}^{\prime}\right)$ : $\left(\mathbf{4}_{F}^{1 / 2}, \mathbf{3}_{S}^{0}\right),\left(\mathbf{4}_{F}^{1 / 2}, \mathbf{5}_{S}^{0}\right),\left(\mathbf{6}_{F}^{1 / 2}, \mathbf{5}_{S}^{0}\right),\left(\mathbf{6}_{F}^{1 / 2}, \mathbf{7}_{S}^{0}\right)$ (fl-1, fl-2, fl-3 and fl-4, respectively). The wiggling lines refer to $A A=H H^{\dagger}$ for $\boldsymbol{R}=\mathbf{4}_{S, F}$, while to $A A=V V(V=W, Z, \gamma)$ for $\boldsymbol{R}=\mathbf{6}_{S, F}$, as required by weak isospin conservation.

figure 2). The lifetime can be estimated according to

$$
\tau_{\mathrm{DM}} \lesssim 2 \times 10^{18}\left(\frac{10^{-2}}{h}\right)^{2}\left(\frac{10^{3} \mathrm{GeV}}{\mu}\right)^{2}\left(\frac{10^{4} \mathrm{GeV}}{m_{\mathrm{DM}}}\right)^{5}\left(\frac{m}{10^{9} \mathrm{GeV}}\right)^{6} \text { sec. } .
$$

Here $h$ refers to a generic Yukawa coupling (see figure 2, diagram (b)). In single messenger scenarios containing $\mathbf{6}_{S, F}^{1 / 2}$, tree level processes involve $\operatorname{dim}=8$ operators (see figure 2, diagrams (c) and (d)). The decay processes will involve two distinct scales, namely $m$ and the scale at which the effective coupling $c_{6}^{S}$ or $c_{7}^{F}$ is generated. This scale can be related with either the scale at which $\alpha_{2}=g_{2}^{2} / 4 \pi^{2}$ becomes nonperturbative or the Planck scale. Depending on the UV completion leading to $c_{6}^{S}$ or $c_{7}^{F}$ the former can be rather low, and thus in some instances $\chi_{\mathrm{DM}}$ decay can be fast. If taken to be $M_{\text {Planck }}$, these processes cannot threaten DM stability. All in all, if one relies only on tree level processes one ends up with the conclusion that scenarios involving representations beyond the quartet (scalar or fermionic) are consistent with DM stability. This conclusion however is misleading since one-loop induced decay processes can always be written and can be fast enough to render $\chi_{\mathrm{DM}}$ cosmologically unstable.

- One-loop level decay modes for $\mathbf{5}_{F}^{0}$. In this case as well one can distinguish single and multiple messenger scenarios, for the former the relevant representations are $\boldsymbol{R}=\mathbf{4}_{S}^{1 / 2}, \mathbf{6}_{S}^{1 / 2}$. These representations enable a $\mathbb{Z}_{2}$-breaking quartic coupling which renders $\chi_{\mathrm{DM}}$ unstable. The corresponding operators are shown in 3 (diagram to the left). These operators lead to different decay modes of which those with no Higgs condensation attachment are dominant, e.g. $\nu W^{ \pm} W^{\mp} Z_{L}$. One can conclude that in one-loop neutrino mass models containing a $4_{S}^{1 / 2}$, DM decays are fast and proceed via tree and one-loop level processes. 
Loop-induced decay processes are of more relevance in models that contain a $\mathbf{6}_{S}^{1 / 2}$. At the tree level these scenarios involve processes that lead to slow DM decay, and so seem consistent with cosmological stability. In the effective limit, the loop-induced processes are associated with $\operatorname{dim}=6$ effective operators whose cutoff scale amounts (at most) to $m=10^{9} \mathrm{GeV}$. Thus, an estimation of the DM lifetime can be done from eq. (2.4) by rescaling by the loop factor. For processes such as $\nu W^{ \pm} W^{\mp} Z_{L}$ the result reads:

$$
\tau_{\mathrm{DM}} \lesssim 7.1 \times 10^{8}\left(\frac{10^{-2}}{\lambda}\right)^{2}\left(\frac{10^{4} \mathrm{GeV}}{m_{\mathrm{DM}}}\right)^{5}\left(\frac{m}{10^{9} \mathrm{GeV}}\right)^{4} \text { sec. . }
$$

Which shows that although the tree level decay does not yield fast decays, the loopinduced processes lead to lifetimes that do not amount to $10^{26}$ seconds, under "reasonable" parameter choices. This conclusion is inline with what was first pointed out in ref. [18], showing the infeasibility of the R $\nu \mathrm{MDM}$ [16]. In the case of multiple messenger fields one finds Yukawa couplings of the type $\boldsymbol{R}_{F}^{1 / 2} \boldsymbol{R}_{F}^{-1 / 2}\left(\boldsymbol{R}^{\prime}\right)_{S}^{0}$, which break the $\mathbb{Z}_{2}$ accidental symmetry. Their presence allow the construction of operators as those shown in figure 3 (diagram to the right), that induce again processes such as $\chi_{\mathrm{DM}} \rightarrow \nu W^{ \pm} W^{\mp} Z_{L}$. The lifetime for these processes amounts to the value of the single messenger case in (2.13).

We now turn to the $\mathbf{7}_{S}^{0}$ DM scenario. As we have already stressed, in the context of minimal DM this representation is not consistent with stability even for $\Lambda=M_{\text {Planck }}$. Thus, even if a one-loop neutrino mass model does not involve degrees of freedom that can generate at the renormalizable level the operator in (2.10) - arguably - quantum gravity effects will generate it and therefore $\varphi_{\mathrm{DM}} \subset \mathbf{7}_{S}^{0}$ will decay at a fast rate [28]. It is however worth identifying those extra representations that enable writing UV completions of this effective operator, in particular because these results will enable in turn the identification of oneloop neutrino mass models where $\varphi_{\mathrm{DM}}$ decays will be due to neutrino physics itself, rather than due to a different type of physics.

As in the fermionic DM scenario, in this case one can identify various $\mathbb{Z}_{2}$-breaking terms whose structure depends on the extra representations present. We have found trilinear scalar couplings of the type $\boldsymbol{7}_{S}^{0} \boldsymbol{R}_{S}^{1 / 2}\left(\boldsymbol{R}_{S}^{\prime}\right)^{-1 / 2}$ and Yukawa couplings $\boldsymbol{7}_{S}^{0} \boldsymbol{R}_{F}^{1 / 2}\left(\boldsymbol{R}_{F}^{\prime}\right)^{-1 / 2}$, with $\boldsymbol{R}=\boldsymbol{R}^{\prime}=\mathbf{6}, \mathbf{8}$ and $\boldsymbol{R}=\mathbf{6}$ and $\boldsymbol{R}^{\prime}=\mathbf{8}$ (for both, fermions and scalars). With these vertices one can then construct a certain number of loop-induced processes that lead to fast $\varphi_{\mathrm{DM}}$ decays. The different operators are shown in figure 4 . The different cases can be sorted in single and multiple messenger scenarios, but due to our universal mass simplification they lead to the same lifetime. Possible decay modes for operators to the left in figure 4 are $W_{L}^{ \pm} W_{L}^{\mp}$, while for those to the right $l^{ \pm} l^{\mp}$. In the effective limit, $m \gg m_{\mathrm{DM}}$, the decay lifetime for processes induced by operators as those shown in the diagram to the left in figure 4 has been calculated as [28]:

$$
\Gamma_{\mathrm{DM}}=\frac{857 C_{0}^{2}}{441548 \pi^{5}} \frac{g^{2} v^{4}}{m^{2} m_{\mathrm{DM}}},
$$




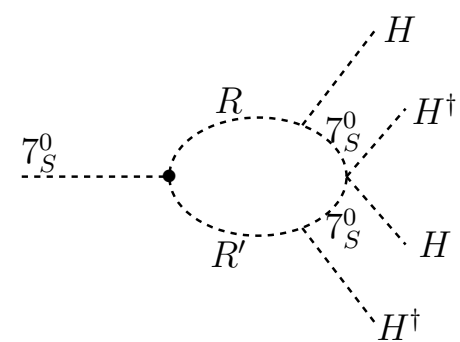

sl-i

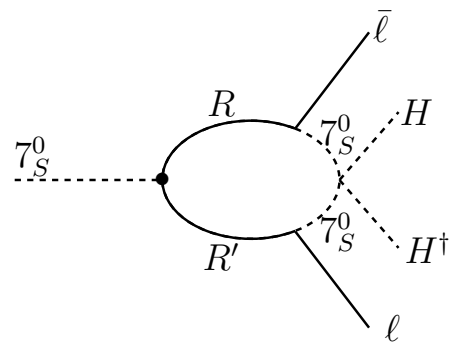

fl-i

Figure 4. Loop-induced operators responsible for $\varphi_{\mathrm{DM}} \subset \boldsymbol{7}_{S}^{0}$ decays. Both diagrams involve three possible operators according to: $R=R^{\prime}=6^{1 / 2}, 8^{1 / 2}$ and $R=6^{1 / 2}$ and $R^{\prime}=8^{1 / 2}$. The label sl-i refers to scalar loops, while fl-i to fermion loops. In both cases, $\mathbf{i}=1$ for $\boldsymbol{R}=\mathbf{6}^{1 / 2}, \mathbf{i}=2$ for $\boldsymbol{R}=\mathbf{6}^{1 / 2}$ and $\boldsymbol{R}^{\prime}=\mathbf{8}^{1 / 2}$ and $\mathbf{i}=3$ for $\boldsymbol{R}=\mathbf{8}^{1 / 2}$. The dot indicates the $\mathbb{Z}_{2}$-breaking interaction.

with $C_{0} \simeq-0.0966$. The lifetime can then be estimated to be

$$
\tau_{\mathrm{DM}} \lesssim 5.9 \times 10^{3}\left(\frac{m_{\mathrm{DM}}}{10^{4} \mathrm{GeV}}\right)\left(\frac{m}{10^{9} \mathrm{GeV}}\right)^{2} \text { sec. } .
$$

For processes with leptons modes (induced by the operators to the right in figure 4) the lifetime is larger, as can be readily understood by realizing that the effective operator is $\operatorname{dim}=6$ rather than 5 , as in the pure scalar case. Though larger, the values are always well below what cosmological stability demands.

\section{UV completions of one-loop realizations of the Weinberg operator}

In order to be model-independent one has to consider all possible one-loop realizations of the lepton-number-breaking $\operatorname{dim}=5$ operator. The possibilities have been systematically sorted in [11], and diagrammatically their number reduces to four independent diagrams as shown in figure 5 . We start by assuming that one of the loop degrees of freedom is either $R=\mathbf{5}_{F}^{0}$ or $R=\mathbf{7}_{S}^{0}$. As soon as this internal representation is fixed the remaining representations are fixed by $\mathrm{SU}(2) \times \mathrm{U}(1)_{Y}$ invariance. It is worth emphasizing that in some instances the resulting representations allow for tree level realizations of higherorder lepton-number-breaking operators (see e.g. ref. [17]). Their contributions to neutrino masses are however negligible provided the BSM fields have masses above $\sim 3 \mathrm{TeV}$.

\subsection{The hypercharge-zero quintet case}

Results for the possible UV completions that contain a hypercharge-zero fermionic quintet are shown in table 1 . The different models can be readily derived with the aid of the $\mathrm{SU}(2)$ product decomposition

$$
\mathbf{2} \otimes \boldsymbol{R}=\boldsymbol{R} \pm 1
$$

and thus rather than listing them one by one we discuss their generic features. A simple inspection to $\mathbf{D} \mathbf{1}$ in figure 5 , shows that fixing $\mathbf{5}_{F}^{0}$ two representations for the internal scalars are possible, namely $\mathbf{4}_{S}^{1 / 2}$ and $\mathbf{6}_{S}^{1 / 2}$. A diagram combining both is of course possible too. 


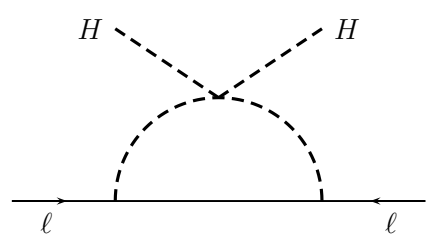

D1

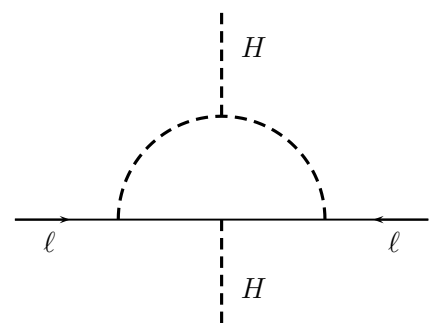

D3

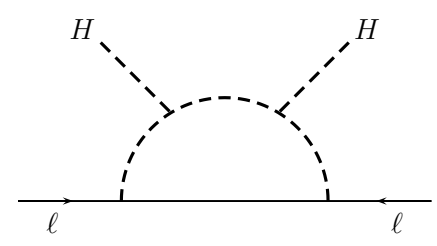

D2

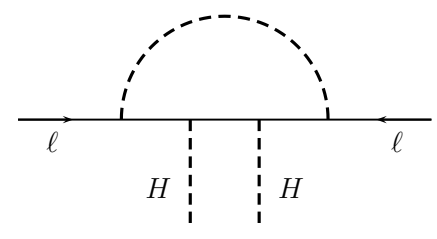

D4

Figure 5. One-loop diagrams for UV realizations of the dim $=5$ Weinberg operator.

In terms of the necessary number of representations, UV completions based on D1 define minimal scenarios. UV completions based on D2 require at least an additional scalar representation, and in contrast to those based on $\mathbf{D} \mathbf{1}$ are such that the representation content always allow for two different contributions to the effective neutrino mass matrix:

$$
m_{\nu}=m_{\nu}^{\mathbf{D} 2}+m_{\nu}^{\mathbf{D} \mathbf{1}}
$$

where $m_{\nu}^{\text {D1 }}$ refers to a contribution to the neutrino mass matrix involving $\mathbf{4}_{S}^{1 / 2}, \mathbf{6}_{S}^{1 / 2}$ or both. Scenarios based on D3 require one extra fermion and two scalar representations. The quantum numbers demanded by gauge invariance are such that the neutrino mass matrix in these cases have always the form

$$
m_{\nu}=m_{\nu}^{\mathbf{D} 3}+m_{\nu}^{\mathbf{D} 2}+m_{\nu}^{\mathbf{D} 1}
$$

regardless of the representations defining D3. UV completions based on D4 are of two types. Those for which the mass matrix is entirely determined by D4 and those that involve a contribution arising from $\mathbf{D} 1$. The former case is obtained whenever in addition to $\mathbf{5}_{F}^{0}$, there is a $\mathbf{4}_{F}^{1 / 2}$ or a $\mathbf{6}_{F}^{1 / 2}$, or both simultaneously. The latter, instead, requires in addition to $\mathbf{4}_{F}^{1 / 2}$ or $\mathbf{6}_{F}^{1 / 2}$ an extra fermionic representation that can be $\mathbf{3}_{F}^{1}, \mathbf{5}_{F}^{1}$ or $\mathbf{7}_{F}^{1}$.

As regards the $\mathbb{Z}_{2}$-breaking decay modes, as can be seen in table 1 all possible models always involve representations that enable writing decay modes as those discussed in section 2.1 (see figure 2-4). In table 1 we have collected models according to the category they belong to, the category defined by diagrams $\mathbf{D 1 - D 4}$, and according to the decay mode they lead to (we have specified only loop-induced processes). Representations yielding a $\mathbb{Z}_{2}$-violating process can be identified from the following color scheme: yellowish (greenish) cells for representations mean that those representations induce a $\mathbb{Z}_{2}$-breaking mode 1 (2). 


\begin{tabular}{|c|c|c|c|c|c|c|c|c|c|c|c|c|}
\hline \multicolumn{10}{|c|}{ Fermion quintet radiative UV completed models } \\
\hline UV & $\mathbf{3}_{F}^{1}$ & $\mathbf{4}_{F}^{1 / 2}$ & $\mathbf{5}_{F}^{1}$ & $\mathbf{6}_{F}^{1 / 2}$ & $\mathbf{7}_{F}^{1}$ & $\mathbf{3}_{S}^{0}$ & $\mathbf{4}_{S}^{1 / 2}$ & $\mathbf{5}_{S}^{0}$ & $\mathbf{6}_{S}^{1 / 2}$ & $\mathbf{7}_{S}^{0}$ & \multicolumn{3}{|c|}{ Decay } \\
\hline D1 & - & - & - & - & - & - & $\checkmark$ & - & $\checkmark$ & - & sl-1 & sl-2 \\
\hline D2 & - & - & - & - & - & $\checkmark$ & $\checkmark$ & $\checkmark$ & $\checkmark$ & $\checkmark$ & sl-1 & sl-2 \\
\hline D3 & - & $\checkmark$ & - & $\checkmark$ & - & $\checkmark$ & $\checkmark$ & $\checkmark$ & $\checkmark$ & $\checkmark$ & fl-i & fl-j \\
\hline D4 & $\checkmark$ & $\checkmark$ & $\checkmark$ & $\checkmark$ & $\checkmark$ & $\checkmark$ & $\checkmark$ & $\checkmark$ & $\checkmark$ & $\checkmark$ & fl-i & sl-j \\
\hline
\end{tabular}

Table 1. One-loop UV completions for models with a fermion quintet defined by diagrams D1-D4 in figure 5. For each diagram and for one of the internal fermions fixed to be $\mathbf{5}_{F}^{0}$, the remaining possible representations are listed according to the notation $\boldsymbol{R}_{a}^{Y}$ (with $\boldsymbol{R}$ referring to the $\mathrm{SU}(2)$ representation, $a=F, S$ and $Y$ to hypercharge). Representations not allowed by gauge invariance are indicated by a dash, while allowed representations are indicated by checkmarks. For the $\mathbb{Z}_{2^{-}}$ breaking decay modes we consider only loop-induced processes. Representations that induce a decay mode are indicated by colors. For example, in the first row $\mathbf{4}_{S}^{1 / 2}$ induces an sl-1 decay processes, while $\mathbf{6}_{S}^{1 / 2}$ an sl-2. For the decay modes see figure 3 . In row 3 (D3), fl-i refers to fl-1 and fl-2, while fl-j to fl-3 and fl-4. The blueish cell refers to a representation "shared" by modes: fl-2 and fl-3. In row 4 (D4), fl-i holds for $\mathrm{i}=1, \ldots, 4$ and sl-j for $\mathbf{j}=1,2$.

\subsection{The hypercharge-zero septet case}

We now turn to the discussion of the scalar septet. As we have already stressed, for this representation there is a loop-induced effective dim $=5$ operator which even in the absence of an UV completion (in "pure" minimal DM) leads to fast DM decay. Despite that, here for completeness we identify the different one-loop neutrino mass models for which UV completions of that effective decay operator can be written.

The main results in this case are summarized in table 2 . The specification of the models in these cases goes along the same lines that in the quintet DM scenarios, with additional $\mathrm{SU}(2)$ product decompositions (e.g. $\mathbf{2} \otimes \mathbf{7}=\mathbf{6} \oplus \mathbf{8}$ ) given by eq. (3.1). With the aid of these product rules the different UV completions, which we do not list, can be easily derived. Their main features are the following. With one of the loop degrees of freedom fixed according to $\mathbf{7}_{S}^{0}$, UV completions based on $\mathbf{D} \mathbf{1}$ are of two types, those involving $\mathbf{6}_{F}^{1}$ and those with $\mathbf{8}_{F}^{1}$. Models relying on $\mathbf{D} 2$ are of two types: models where the mass matrix is entirely determined by D2-type contributions and scenarios where the neutrino mass matrix has the form:

$$
m_{\nu}=m_{\nu}^{\mathbf{D} 2}+m_{\nu}^{\mathbf{D} 1}
$$

In D3-based scenarios the representations required imply in all cases a neutrino mass matrix of the form

$$
m_{\nu}=m_{\nu}^{\mathbf{D} 3}+m_{\nu}^{\mathbf{D} 2}+m_{\nu}^{\mathbf{D} 1} .
$$

Finally, for UV completions involving D4 diagrams the neutrino mass matrix involves only D4 contributions. 


\begin{tabular}{|c|c|c|c|c|c|c|c|c|c|c|c|c|}
\hline \multicolumn{10}{|c|}{ Scalar septet radiative UV completed models } \\
\hline UV & $\mathbf{5}_{F}^{0}$ & $\mathbf{6}_{F}^{1 / 2}$ & $\mathbf{7}_{F}^{0}$ & $\mathbf{8}_{F}^{1 / 2}$ & $\mathbf{9}_{F}^{0}$ & $\mathbf{5}_{S}^{1}$ & $\mathbf{6}_{S}^{1 / 2}$ & $\mathbf{7}_{S}^{1}$ & $\mathbf{8}_{S}^{1 / 2}$ & $\mathbf{9}_{S}^{1}$ & \multicolumn{3}{|c|}{ Decay } \\
\hline D1 & - & $\checkmark$ & - & $\checkmark$ & - & $\checkmark$ & - & $\checkmark$ & - & $\checkmark$ & Mode 1 & Mode 2 \\
\hline D2 & $\checkmark$ & $\checkmark$ & $\checkmark$ & $\checkmark$ & $\checkmark$ & $\checkmark$ & $\checkmark$ & $\checkmark$ & $\checkmark$ & $\checkmark$ & sl-1 & sl-3 \\
\hline D3 & $\checkmark$ & $\checkmark$ & $\checkmark$ & $\checkmark$ & $\checkmark$ & - & $\checkmark$ & - & $\checkmark$ & - & $\mathrm{fl}-\mathbf{1}$ & sl-j \\
\hline D4 & $\checkmark$ & $\checkmark$ & $\checkmark$ & $\checkmark$ & $\checkmark$ & - & - & - & - & - & $\mathrm{fl}-1$ & $\mathrm{fl}-3$ \\
\hline
\end{tabular}

Table 2. One-loop UV completions for models with a scalar septet defined by diagrams D1-D4 in figure 5. For each diagram and for one of the internal scalars fixed to be $\boldsymbol{7}_{S}^{0}$, the remaining possible representations are listed according to the notation $\boldsymbol{R}_{a}^{Y}$ (with $\boldsymbol{R}$ referring to the $\mathrm{SU}(2)$ representation, $a=F, S$ and $Y$ to hypercharge). Representations not allowed by gauge invariance are indicated by a dash, while allowed representations are indicated by checkmarks. For the $\mathbb{Z}_{2^{-}}$ breaking decay modes we consider only loop-induced processes. Representations that induce a decay mode are indicated by colors. For example, in the first row $\mathbf{6}_{F}^{1 / 2}$ induces an fl-1 decay processes, while $\mathbf{8}_{F}^{1 / 2}$ an fl-3. For the different decay modes see figure 4 . In row 3 (D3), fl-i refers to fl-1 and fl-3, while sl-j to sl-1 and sl-3.

Regarding $\mathbb{Z}_{2}$-breaking modes, as shown in table 2 all UV completions of one-loop realizations of the Weinberg operator always involve a $\mathbb{Z}_{2}$-violating decay mode. As we have pointed out in section 2.1, compared with the fermion quintet these processes lead to faster DM decay since they are related with $\operatorname{dim}=5$ decay operators. The color scheme follows the same conventions of table 1: yellowish (greenish) cells for representations mean that those representations induce a $\mathbb{Z}_{2}$-breaking mode $1(2)$, with the different modes referring to figure 4 .

\section{Pitfalls of DM from higher-order representations and neutrino masses}

The different decay modes we have identified depend upon neutrino-related parameters and $\mathbb{Z}_{2}$-breaking couplings. In the estimations of lifetimes we have taken the former to their extreme values, while keeping perturbative Yukawa couplings $\left(\left|Y_{\nu}\right|<1\right)$. Thus, if one takes the extra representations to be heavier the largest neutrino mass will be below $m_{\mathrm{Atm}}^{\operatorname{Exp}} \simeq 0.05 \mathrm{eV}$. The only way that enables the increasing of the lifetime is through couplings not related with neutrino physics that can be either $\mathbb{Z}_{2}$-breaking or $\mathbb{Z}_{2}$-conserving. For neutrino-related parameters taken to their extreme values, couplings of order $10^{-11}$ or so (see e.g. eq. (2.13)) will lead to lifetimes of order $10^{26}$ seconds. Stability, however, is not assured anymore by an accidental symmetry but from the smallness of a parameter. Whether such small coupling is justified depends on the UV completed model, e.g. it could be that in some UV realizations the coupling is radiatively induced or generated from the spontaneous symmetry breaking of a symmetry present in the UV completed theory (it has a dynamical origin ${ }^{7}$ ), case in which a small value would be justified. One could as well

\footnotetext{
${ }^{7}$ For models of cosmological stable DM with dynamically generated small couplings see e.g. [40].
} 
argue that for $\mathbb{Z}_{2}$-breaking couplings their smallness is technically "natural", in 't Hooft's sense [41], since tiny values increase the symmetry of the Lagrangian. This statement, however, depends on their RGE stability and therefore on their nature. For $\mathbb{Z}_{2}$-breaking Yukawas, diagrams contributing to RGE running are proportional to the Yukawa itself (or certain power) and so one expects their values to be RGE stable. For scalar quartic couplings, instead, this is not necessarily the case and strongly depends on the dimension of the representations involved [42]. Thus, in the absence of an explicit UV completed model DM slow decays are probably better justified if they arise from small Yukawas.

Note that even smaller couplings are required if one lowers the mass scale of the messenger fields. The point is that with $m \sim 10^{9} \mathrm{GeV}$ any possible low-energy indirect probe (e.g. lepton-flavor-violating or collider-related observables) is well below current and future experimental sensitivities. Thus, testability of these scenarios requires $m \ll 10^{9} \mathrm{GeV}$. For scales comparable to those of DM the lifetimes are reduced by several order of magnitudes and so smaller couplings, order $10^{-21}$ or so, are required to achieve consistent lifetimes. Thus, in summary, in neutrino mass models that contain extra representations as those we have identified in the previous section (in addition to $\mathbf{5}_{F}^{0}$ or $\mathbf{7}_{S}^{0}$, or both) one expects DM to decay fast, with the decays determined by $\mathbb{Z}_{2}$-violating operators (see figures $2-4$ ). The explicit breaking of the accidental $\mathbb{Z}_{2}$ symmetry renders DM cosmologically unstable. Tuning of couplings is possible, but in that case cosmological stability is unrelated with the mechanism underlying minimal DM models.

A more plausible way of relating Majorana neutrino masses with accidentally stabilized $\mathrm{DM}$ is through higher-order loops. In this case, $\mathrm{SU}(2) \times \mathrm{U}(1)_{Y}$ invariance is less restrictive due to the topologies of the possible diagrams. In the two-loop case, the topologies allow for overall shifts in the hypercharge of the fields flowing in the loops, while keeping $Y=0$ for the DM representation (for the different topologies see ref. [12]). This "hypercharge freedom" might enable the construction of consistent two-loop models. For three-loop models, as far as we are aware, two examples have been pointed out [22, 23]. Ref. [22] considers a model with three $\mathbf{7}_{F}^{0}$, one $\mathbf{7}_{S}^{1}$ and one $\mathbf{1}_{S}^{1}$. The neutral component of the lightest $\mathbf{7}_{F}^{0}$ is cosmologically stable due to an accidental $\mathbb{Z}_{2}$ symmetry. Ref. [23], instead, considers a model with three copies of the $\mathbf{5}_{F}^{0}$, one copy of the $\boldsymbol{7}_{S}^{0}$ and one copy of the $\boldsymbol{5}_{S}^{1}$, in addition to a two-Higgs-doublet model. In this case, the neutral component of the lightest $\mathbf{5}_{F}^{0}$ is cosmologically stable. Although consistent with DM stability, neutrino physics data and low-energy lepton-flavor-violating constraints, we have found that in both cases $\alpha_{2}=g_{2}^{2} / 4 \pi$ reaches a Landau pole at relatively low energy scales: $\Lambda_{\text {Landau-pole }}^{(1)} \simeq 10 m_{X}, \Lambda_{\text {Landau-pole }}^{(2)} \simeq$ $10^{2} m_{X}$ (see figure 6), where $m_{X}$ refers to the masses of the heavy representations which we have taken to be universal, for simplicity. This result has been derived by integrating two-loop RGEs and neglecting Yukawa couplings contributions. Note that since in threeloop models Yukawas are order one, their inclusion will change the location of the Landau pole, with the precise value increasing or decreasing depending on numerical details.

This result then shows that in these scenarios perturbativity is lost at scales a couple orders of magnitude higher from the characteristic energy scale of the models (taken to be $m_{X}$ ), with such behavior being more pronounced for the model in [22]. In the absence of a strongly coupled $\mathrm{SU}(2) \times \mathrm{U}(1)_{Y}$ sector at that scale, ideally one should demand pertur- 

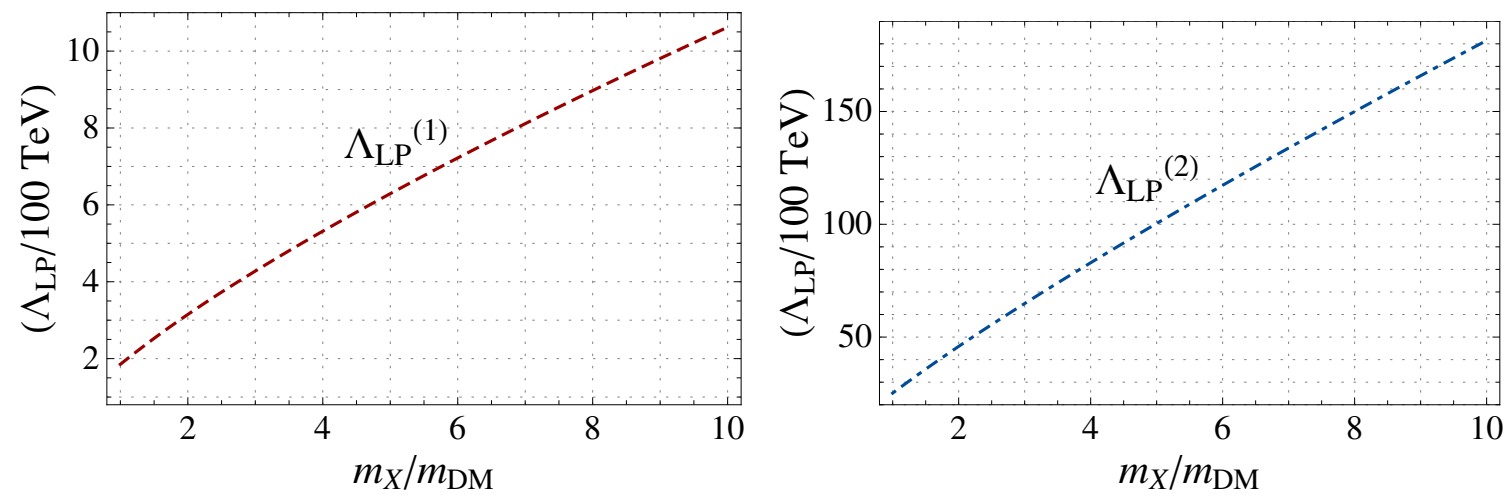

Figure 6. Energy scale at which $\alpha_{2}$ reaches a Landau pole as a function of $m_{X} / m_{\mathrm{DM}}$ in the three-loop neutrino models of $[22,23]$ ( $m_{X}$ refers to the masses of the heavier fermion and scalars). The plot to the left is the result for the model in [22] with the DM mass fixed to $20 \mathrm{TeV}$ and the singlet scalar $\left(\mathbf{1}_{S}^{1}\right)$ mass to $500 \mathrm{GeV}$. The plot to the right is for [23] with the DM mass fixed to $10 \mathrm{TeV}$ and the second doublet mass to $1 \mathrm{TeV}$. These results have been derived from two-loop RGEs and neglecting Yukawa contributions [43] (see text for more details).

bativity to hold at least up to the GUT scale, where one could expect that a new gauge sector assures $\alpha_{2}<1$. Thus, arguably, this may be a drawback of these approaches. The presence of multiple higher-order representations can lead to non-perturbative scenarios at relatively low energy scales. There is of course something one should bear in mind. One could in principle understand these scenarios as effective limits of a larger UV theory, in which it could be that by taken into account all the degrees of freedom and intercations perturbativity could be restored. Furthermore, in that theory it could be as well that the $\mathbb{Z}_{2}$-violating couplings that we have identified as responsible for DM fast decay are either absent or dynamically suppressed, case in which DM will become cosmologically stable.

\section{Conclusions}

In this paper, we have presented a model-independent study of one-loop neutrino mass models in which one of the loop messengers is a hypercharge-zero fermion quintet or a hypercharge-zero scalar septet. In the absence of additional higher-order EW representations the neutral component of the quintet is absolutely stable at the renormalizable level, and cosmologically stable when the leading-order effective operator responsible for its decay $(\operatorname{dim}=6)$ is included (assuming a cutoff above $10^{15} \mathrm{GeV}$, see figure 1 ). We have shown that such feature no longer holds in the presence of the extra degrees of freedom that define the one-loop neutrino mass model, regardless of the model. For completeness we have extended our analysis to include the case of a hypercharge-zero scalar septet, although this representation cannot be reconciled with cosmological stability even in minimal DM scenarios.

We have systematically classified all such models and the corresponding DM decay operators in each case. Our main findings are summarized in tables 1 and 2, combined with the different DM decay operators given in figures 3 and 4 . From these results our main 
conclusion is that one-loop neutrino mass models that use minimal DM representations are not consistent with DM stability, unless tiny couplings $-\mathcal{O} \sim 10^{-21}-10^{-11}$ (with the value depending on the highest scale of the corresponding neutrino mass model) — or ad hoc symmetries are assumed. The former being hard to reconcile in the absence of a dynamical mechanism assuring such extreme small values, while the latter missing the whole point behind higher-order EW representations. ${ }^{8}$

We have commented on neutrino mass models variations based on higher-order loops. We argued that in the two-loop case, models consistent with DM stability might be possible writing due to the "hypercharge freedom" that the two-loop topologies offer. We have stressed that these models and three-loop scenarios might be hard to reconcile with perturbativity criteria. We have illustrated that for two specific three-loop models for which we have found that $\alpha_{2}$ reaches a Landau pole at relatively low energy scales (figure 6), $\Lambda_{\text {Landau-pole }} \sim\left(10-10^{2}\right) m_{X}$, with $m_{X}$ the characteristic scale of the model.

\section{Acknowledgments}

We would like to thank Sofiane Boucenna, Thomas Hambye, Alejandro Ibarra, Igor Ivanov and Luca di Luzio for useful comments. We specially thank Michael Schmidt and Yi Cai for useful email exchanges and for sharing with us results of their work [21] prior to its submission to arXiv. This work was supported by the "Fonds de la Recherche ScientifiqueFNRS" under grant number 4.4501.15. The work of C.S. is supported by the "Université de Liège" and the EU in the context of the MSCA-COFUND-BeIPD project.

Note added in proof. While completing this work, two papers reaching conclusions similar to ours appeared [21, 24].

Open Access. This article is distributed under the terms of the Creative Commons Attribution License (CC-BY 4.0), which permits any use, distribution and reproduction in any medium, provided the original author(s) and source are credited.

\section{References}

[1] S. Weinberg, Baryon and lepton nonconserving processes, Phys. Rev. Lett. 43 (1979) 1566 [INSPIRE].

[2] Particle Data Group collaboration, K.A. Olive et al., Review of particle physics, Chin. Phys. C 38 (2014) 090001 [inSPIRE].

[3] D. Aristizabal Sierra, A. Degee and J.F. Kamenik, Minimal lepton flavor violating realizations of minimal seesaw models, JHEP 07 (2012) 135 [arXiv:1205.5547] [INSPIRE].

[4] J.L. Feng, Dark matter candidates from particle physics and methods of detection, Ann. Rev. Astron. Astrophys. 48 (2010) 495 [arXiv: 1003.0904] [INSPIRE].

[5] E. Ma, Verifiable radiative seesaw mechanism of neutrino mass and dark matter, Phys. Rev. D 73 (2006) 077301 [hep-ph/0601225] [INSPIRE].

\footnotetext{
${ }^{8}$ From the cosmological point of view, these representations are motivated for they provide stabilization without the need of additional symmetries.
} 
[6] D. Aristizabal Sierra, J. Kubo, D. Restrepo, D. Suematsu and O. Zapata, Radiative seesaw: warm dark matter, collider and lepton flavour violating signals,

Phys. Rev. D 79 (2009) 013011 [arXiv: 0808.3340] [INSPIRE].

[7] S. Davidson, E. Nardi and Y. Nir, Leptogenesis, Phys. Rept. 466 (2008) 105 [arXiv:0802.2962] [INSPIRE].

[8] C.S. Fong, E. Nardi and A. Riotto, Leptogenesis in the universe, Adv. High Energy Phys. 2012 (2012) 158303 [arXiv:1301.3062] [INSPIRE].

[9] D. Aristizabal Sierra, M. Losada and E. Nardi, Variations on leptogenesis, Phys. Lett. B 659 (2008) 328 [arXiv:0705.1489] [INSPIRE].

[10] D. Aristizabal Sierra, L.A. Muñoz and E. Nardi, Purely flavored leptogenesis, Phys. Rev. D 80 (2009) 016007 [arXiv:0904.3043] [inSPIRE].

[11] F. Bonnet, M. Hirsch, T. Ota and W. Winter, Systematic study of the $d=5$ Weinberg operator at one-loop order, JHEP 07 (2012) 153 [arXiv:1204.5862] [INSPIRE].

[12] D. Aristizabal Sierra, A. Degee, L. Dorame and M. Hirsch, Systematic classification of two-loop realizations of the Weinberg operator, JHEP 03 (2015) 040 [arXiv:1411.7038] [INSPIRE].

[13] M. Kadastik, K. Kannike and M. Raidal, Dark matter as the signal of grand unification, Phys. Rev. D 80 (2009) 085020 [Erratum ibid. D 81 (2010) 029903] [arXiv:0907.1894] [INSPIRE].

[14] M. Frigerio and T. Hambye, Dark matter stability and unification without supersymmetry, Phys. Rev. D 81 (2010) 075002 [arXiv:0912.1545] [inSPIRE].

[15] D. Aristizabal Sierra, M. Dhen, C.S. Fong and A. Vicente, Dynamical flavor origin of $\mathbb{Z}_{N}$ symmetries, Phys. Rev. D 91 (2015) 096004 [arXiv: 1412.5600] [INSPIRE].

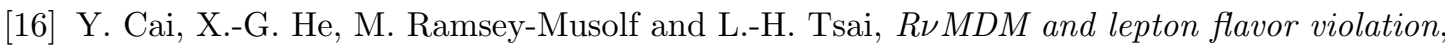
JHEP 12 (2011) 054 [arXiv: 1108.0969] [INSPIRE].

[17] K. Kumerički, I. Picek and B. Radovčić, TeV-scale seesaw with quintuplet fermions, Phys. Rev. D 86 (2012) 013006 [arXiv:1204.6599] [inSPIRE].

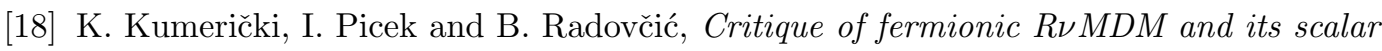
variants, JHEP 07 (2012) 039 [arXiv:1204.6597] [INSPIRE].

[19] S.S.C. Law and K.L. McDonald, A class of inert $N$-tuplet models with radiative neutrino mass and dark matter, JHEP 09 (2013) 092 [arXiv: 1305.6467] [INSPIRE].

[20] C.-H. Chen and S.S.C. Law, Exotic fermion multiplets as a solution to baryon asymmetry, dark matter and neutrino masses, Phys. Rev. D 85 (2012) 055012 [arXiv:1111.5462] [INSPIRE].

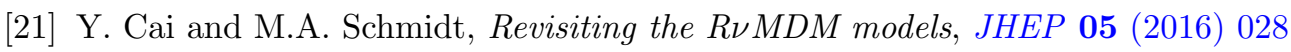
[arXiv: 1603.00255] [INSPIRE].

[22] A. Ahriche, K.L. McDonald, S. Nasri and T. Toma, A model of neutrino mass and dark matter with an accidental symmetry, Phys. Lett. B 746 (2015) 430 [arXiv: 1504.05755] [INSPIRE].

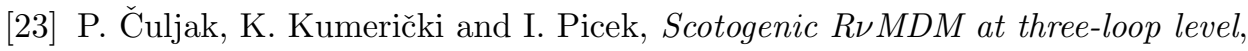
Phys. Lett. B 744 (2015) 237 [arXiv:1502.07887] [INSPIRE].

[24] A. Ahriche, K.L. McDonald, S. Nasri and I. Picek, A critical analysis of one-loop neutrino mass models with minimal dark matter, Phys. Lett. B 757 (2016) 399 [arXiv:1603. 01247] [INSPIRE]. 
[25] M. Cirelli, N. Fornengo and A. Strumia, Minimal dark matter, Nucl. Phys. B 753 (2006) 178 [hep-ph/0512090] [INSPIRE].

[26] M. Cirelli, A. Strumia and M. Tamburini, Cosmology and astrophysics of minimal dark matter, Nucl. Phys. B 787 (2007) 152 [arXiv:0706.4071] [INSPIRE].

[27] M. Cirelli and A. Strumia, Minimal dark matter: model and results, New J. Phys. 11 (2009) 105005 [arXiv:0903.3381] [InSPIRE].

[28] L. Di Luzio, R. Gröber, J.F. Kamenik and M. Nardecchia, Accidental matter at the LHC, JHEP 07 (2015) 074 [arXiv: 1504.00359] [INSPIRE].

[29] E. Del Nobile, M. Nardecchia and P. Panci, Millicharge or decay: a critical take on minimal dark matter, JCAP 04 (2016) 048 [arXiv: 1512.05353] [INSPIRE].

[30] M. Cirelli, T. Hambye, P. Panci, F. Sala and M. Taoso, Gamma ray tests of minimal dark matter, JCAP 10 (2015) 026 [arXiv:1507.05519] [INSPIRE].

[31] C. Garcia-Cely, A. Ibarra, A.S. Lamperstorfer and M.H.G. Tytgat, Gamma-rays from heavy minimal dark matter, JCAP 10 (2015) 058 [arXiv:1507.05536] [INSPIRE].

[32] F. Wilczek and A. Zee, Operator analysis of nucleon decay, Phys. Rev. Lett. 43 (1979) 1571 [INSPIRE].

[33] S. Ando and K. Ishiwata, Constraints on decaying dark matter from the extragalactic gamma-ray background, JCAP 05 (2015) 024 [arXiv: 1502.02007] [INSPIRE].

[34] C. Rott, K. Kohri and S.C. Park, Superheavy dark matter and IceCube neutrino signals: bounds on decaying dark matter, Phys. Rev. D 92 (2015) 023529 [arXiv:1408.4575] [INSPIRE].

[35] A. Ibarra, A.S. Lamperstorfer and J. Silk, Dark matter annihilations and decays after the AMS-02 positron measurements, Phys. Rev. D 89 (2014) 063539 [arXiv:1309.2570] [INSPIRE].

[36] G. Giesen et al., AMS-02 antiprotons, at last! Secondary astrophysical component and immediate implications for dark matter, JCAP 09 (2015) 023 [arXiv:1504.04276] [INSPIRE].

[37] D.V. Forero, M. Tortola and J.W.F. Valle, Neutrino oscillations refitted, Phys. Rev. D 90 (2014) 093006 [arXiv: 1405.7540] [InSPIRE].

[38] M.C. Gonzalez-Garcia, M. Maltoni and T. Schwetz, Updated fit to three neutrino mixing: status of leptonic CP-violation, JHEP 11 (2014) 052 [arXiv:1409.5439] [INSPIRE].

[39] F. Capozzi et al., Status of three-neutrino oscillation parameters, circa 2013, Phys. Rev. D 89 (2014) 093018 [arXiv:1312.2878] [InSPIRE].

[40] D. Aristizabal Sierra, D. Restrepo and O. Zapata, Decaying neutralino dark matter in anomalous $\mathrm{U}(1)_{H}$ models, Phys. Rev. D 80 (2009) 055010 [arXiv: 0907.0682] [INSPIRE].

[41] G. 't Hooft, Naturalness, chiral symmetry, and spontaneous chiral symmetry breaking, NATO Sci. Ser. B 59 (1980) 135 [InSPIRE].

[42] Y. Hamada, K. Kawana and K. Tsumura, Landau pole in the standard model with weakly interacting scalar fields, Phys. Lett. B 747 (2015) 238 [arXiv:1505.01721] [INSPIRE].

[43] M.E. Machacek and M.T. Vaughn, Two-loop renormalization group equations in a general quantum field theory: I. Wave function renormalization, Nucl. Phys. B 222 (1983) 83 [INSPIRE]. 